\title{
On the dynamic detonation parameters in acetylene-oxygen mixtures with varying amount of argon dilution
}

Bo Zhang ${ }^{1,2 \dagger}$, Navid Mehrjoo ${ }^{3}$, Hoi Dick Ng${ }^{3}$, John H.S. Lee ${ }^{4}$, Chunhua Bai ${ }^{2}$

${ }^{1}$ East China University of Science and Technology

State Environmental Protection Key Laboratory of Risk Assessment and Control on Chemical Process, Shanghai, 200237, China

${ }^{2}$ Beijing Institute of Technology

State Key Laboratory of Explosion Science and Technology, Beijing, 100081, China

${ }^{3}$ Concordia University

Department of Mechanical and Industrial Engineering

Montréal, H3G 1M8, Canada

${ }^{4}$ McGill University

Department of Mechanical Engineering, Montréal, H3A 2K6, Canada

${ }^{\dagger}$ Corresponding Author

East China University of Science and Technology

State Environmental Protection Key Laboratory of Risk Assessment and Control on Chemical Process Shanghai 200237, China

E-mail: bzhang@ecust.edu.cn

Tel.: (86) 21-64253132

Fax: (86) 21-64253132 


\title{
On the dynamic detonation parameters in acetylene-oxygen mixtures with varying amount of argon dilution \\ by
}

\author{
Bo Zhang, Navid Mehrjoo, Hoi Dick Ng, John H.S. Lee, Chunhua Bai
}

\begin{abstract}
In this investigation, the dynamic detonation parameters for stoichiometric acetylene-oxygen mixtures diluted with varying amount of argon are measured and analyzed. The experimental results show that the critical tube diameter and the critical energy for direct initiation of spherical detonations increase with the increase of argon dilution. The scaling behavior between the critical tube diameter $d_{c}$ and the detonation cell size $\lambda$ as well as the critical direct initiation energy $E_{\mathrm{c}}$ is systematically studied with the effect of argon dilution. The present results again validate that the relation $d_{c}=13 \lambda$ holds for $0 \%-30 \%$ argon diluted mixtures and breaks down when argon dilution increases up to $40 \%$. It is found that the explosion length scaling of $R_{0} \sim 26 \lambda$ becomes also invalid when the mixture contains approximately this same amount of argon dilution or more. This critical argon dilution is indeed close to that found from experiments in porous-walled tubes by Radulescu and Lee (2002) which exhibit a distinct transition in the failure mechanism. Cell size analysis in literature also indicates that the cellular detonation front starts to become more regular (or stable) when the argon dilution reaches more than 40 - 50\%. Regardless of the degree of argon dilution or mixture sensitivity, the phenomenological model developed from the surface energy concept by Lee, which provides a relation that links the critical tube diameter and the critical energy remains valid. The present experimental results also follow qualitatively the observation from chemical kinetic and detonation instability analyses.
\end{abstract}

Keywords: Detonation; Critical tube diameter; Critical initiation energy; Cell size; Argon dilution 


\section{Introduction}

Gaseous detonations in most hydrocarbon mixtures are generally unstable with an ensemble of transverse waves interacting at the shock front that forms the characteristic irregular cellular structure [1]. In critical situations where the detonation propagation is close to failure, or with initial and mixture conditions at which the detonation characteristic cell size becomes comparable with the physical boundary size and dependent on these conditions, the instability at the cellular front can play an important major role on the dynamics of the detonation wave. Lee [1] argued that for an unstable detonation particularly at the above scenarios, the inability to maintain or develop instability at the detonation front is the mechanism that leads to failure in several situations. For example, detonation waves are observed to fail when the instabilities are suppressed as transverse waves are damped by acoustic absorbing walls or porous media [2-4]. Studies also suggested that detonation limits in tubes [5] and the critical tube diameter problem [6] are due to a local failure mechanism where instabilities fail to be maintained at the wave front. For a self-sustained diverging detonation, new cells must also be continuously generated via instability as the wave expands, otherwise the unstable diverging cellular detonation may fail to propagate outward in the radial direction [1]. Hence, both favorable initial conditions and detonation instability are essential and bring together mechanisms to promote sufficiently high combustion rates that can maintain the propagation of a self-sustained detonation.

Using the detonation cell size $\lambda$ to characterize the unstable cellular structure, dynamic parameters in these common mixtures usually follow well with classical empirical correlations. For example, the critical tube diameter scales with the detonation cell size according to the $d_{\mathrm{c}}=13 \lambda$ correlation $[7,8]$. The critical energy for direct initiation of spherical detonations $[9,10]$ can also be scaled adequately from the explosion length $R_{0} \sim 26 \lambda$ where $R_{0}=\left(E_{\mathrm{c}} / p_{0}\right)^{1 / 3}$. Exceptions to these universal correlations were mixtures with high argon dilution [11-13] that emerged from smoked foil measurements, the 
corresponding detonation structure is usually found to be highly regular or piece-wise laminar closely described by the classical Zel’dovich-von Neumann-Döring (ZND) model [11-14].

Highly argon diluted mixtures were often considered as special mixtures to investigate the dynamics of detonation initiation and propagation, failure in detonation limits and the critical tube diameter problem (e.g., [4, 12-17]). In highly argon diluted mixtures, the propagation relies mainly on the shock ignition mechanism and the ZND induction length scale represents an appropriate chemical length to correlate with different dynamic detonation parameters [10, 15]. Detonation limits in tubes and the transmission of a detonation wave from a confined tube into a sudden open area are also thought to be governed by a global failure mechanism. This mechanism appears to be driven from excessive front curvature, above a critical value of which a steady ZND detonation can no longer be obtained $[5,18$, 19]. Evidences also pointed out that the local instability seems not to play a prominent role in the critical tube diameter problem [6].

To clarify between the two possible modes of propagation and failure mechanism, i.e., one caused by suppression of instability and the other by excessive curvature, previous studies often considered the two extreme cases, i.e., detonations in undiluted $\mathrm{C}_{2} \mathrm{H}_{2}-\mathrm{O}_{2}$ mixtures and diluted $\mathrm{C}_{2} \mathrm{H}_{2}-\mathrm{O}_{2}$ mixtures with heavy amount of argon addition more than $70 \%$. Only few studies systematically investigate the quantitative effect of increasing amount of argon dilution on the behavior of the detonation wave and its dynamic parameters. It is of interest not only to look at the transition of the two proposed distinct modes of propagation and failure mechanism, but also to study different scaling relationships and to determine what quantity of argon diluent in the explosive mixture such that cellular instabilities start to become less significant in the detonation dynamics.

In this study, the critical tube diameter and critical energy of direct initiation of spherical detonations in stoichiometric acetylene-oxygen mixtures diluted with varying amount of argon from $0 \%$ to $70 \%$ at 
different initial pressures are measured experimentally. New experimental data of critical tube diameter and critical initiation energy are first reported, and the relation between these detonation dynamic parameters along with increasing amount of argon dilution in stoichiometric acetylene-oxygen mixtures is then discussed.

\section{Experimental Details}

Mixtures of stoichiometric $\mathrm{C}_{2} \mathrm{H}_{2}-\mathrm{O}_{2}$ with different argon dilutions were investigated; the sensitivity of the mixtures was controlled by the initial pressure $p_{0}$ and the range is given in Table 1 . The mixture were prepared beforehand in a separate vessel by the method of partial pressure and the gases were allowed to mix for at least 24 hours to ensure homogeneity for each tested mixture. Schematics of the experimental apparatus for the measurements of the critical tube diameter and critical energy are shown in Fig. 1. Both experimental setups were previously used for the same type of measurement for other hydrocarbon systems (e.g., $[10,15,20,21])$ and therefore, detailed description is omitted here.

For the critical tube diameter measurement, the apparatus is shown in Fig.1a. The setup connected a vertical circular steel tube to a large high-pressure spherical chamber. Different diameters of the tube $d$ were considered, i.e., $d=19.1,15.5,12.7$ and $9.1 \mathrm{~mm}$. The tube diameter $d$ was varied via inserting smaller diameter tubes. The detonation was initiated at the top of the vertical circular steel tube and subsequently transmitted into the relatively larger spherical chamber. A photo probe and a piezoelectric shock pin (CA-1136, Dynasen Inc.) were mounted at the top and bottom of the spherical bomb, which were used to record the time-of-arrival (TOA) signals of the wave. From the TOA between initiation and photo probe - which locates at the top of the spherical bomb (i.e., near the end of the vertical tube) - it

can be known whether a successful detonation is first initiated in the vertical tube. Using the TOA measurement from the piezoelectric shock pin located at the bottom of the spherical chamber, it is then 
possible to distinguish between successful detonation transmission or failure. For example, successful transmission and failure cases in a stoichiometric $\mathrm{C}_{2} \mathrm{H}_{2}-\mathrm{O}_{2}$ mixture with the tube diameter of $19.05 \mathrm{~mm}$ and initial pressures of $p_{0}=12 \mathrm{kPa}$ and $11 \mathrm{kPa}$ are shown in Fig. 2 and Fig. 3, respectively. It can be seen from Fig. 2 that at an initial pressure of $12 \mathrm{kPa}$, the arrival time of the expanding wave is $201 \mu \mathrm{sec}$ when it reaches the photo probe and $317 \mu$ sec at the shock pin. The computed velocities of the wave are $2073.4 \mathrm{~m} / \mathrm{s}$ and $2136.7 \mathrm{~m} / \mathrm{s}$ in the vertical tube and spherical chamber, which are $91.1 \%$ and $94.4 \%$ of the CJ detonation velocity, respectively. It shows that at an initial pressure of $12 \mathrm{kPa}$, the tube diameter is above the critical value, thus the planar detonation can successfully transit into a spherical detonation. While for an unsuccessful transmission, Fig. 3 shows that when the initial pressure decreases to $11 \mathrm{kPa}$, although a detonation wave propagates in the vertical tube at a velocity around $90 \% \mathrm{CJ}$ detonation velocity, the detonation fails after exiting into the free space and the velocity of the expanding wave is only $23.6 \%$ of the CJ velocity value. Validation and further details can be found in [22, 25].

For the direct initiation experiment, measurements were carried in the same high-pressure spherical chamber of $20.3 \mathrm{~cm}$ in diameter and $5.1 \mathrm{~cm}$ in wall thickness, which is shown in Fig.1b. The direct detonation initiation of a spherical detonation was achieved via a high voltage spark discharge from an ignition circuit. The energy was delivered through the end of a slender electrode with a $3.5 \mathrm{~mm}$ spark gap located at the top of the spherical chamber. The initiation event was recorded by the signal from a PCB piezoelectric pressure transducer. The procedure to distinguish detonation initiation or failure is similar to the procedure described above for the critical tube diameter experiments using the TOA measurement recorded by the PCB pressure transducer, and details to estimate the actual spark discharge energy from the ignition system can be found in authors' previous studies [10, 15, 20-25]. Both the critical tube diameter and critical energy measurement experiment were performed at the initial temperature of $293 \mathrm{~K}$. 


\section{Results and Discussion}

Figure 4 first shows the experimental results and curve fits of critical tube diameter as a function of critical pressure for varying degree of argon dilution from $0 \%$ to $70 \%$ in stoichiometric acetyleneoxygen mixtures. The critical initial pressure is measured and is defined as the condition below which the emergent planar detonation from the vertical steel tube fails to transmit into large spherical chamber. It can be seen from Fig. 4 that the critical pressure increases consequently with increasing amount of argon dilution at the same tube diameter. Equivalently, this result indicates that the detonation is less sensitive with larger amount argon dilution. As one can deduce intuitively, the critical pressure value is also shown to be lower when a circular tube with larger diameter $d$ is used for the same mixture.

The critical initiation energies for direct detonation initiation at each initial pressure are also summarized and shown in Fig. 5 for $\mathrm{C}_{2} \mathrm{H}_{2}+2.5 \mathrm{O}_{2}$ mixtures with various degrees of argon dilution. It can be observed that the effect of increasing argon dilution on the critical energy is very similar to that on the critical tube diameter, i.e., the more amount of argon in the stoichiometric $\mathrm{C}_{2} \mathrm{H}_{2}+2.5 \mathrm{O}_{2}$ mixture, the larger the initiation energy is required to form directly a spherical detonation.

From the present results of the critical tube diameter and critical energy for direct initiation of detonations in $\mathrm{C}_{2} \mathrm{H}_{2}+2.5 \mathrm{O}_{2}+\%$ Ar mixtures, it is shown that the effect of increasing argon dilution leads to higher values of these two dynamic detonation parameters. In other words, high argon diluted acetylene-oxygen detonations are more susceptible to failure and harder to initiate. To further analyze the present experiment data, it is of interest to investigate the scaling between the cell size $\lambda$, critical tube diameter $d_{\mathrm{c}}$ and critical initiation energy $E_{\mathrm{c}}$ in mixtures diluted with different amounts of argon. Part of the required cell size data for $\mathrm{C}_{2} \mathrm{H}_{2}+2.5 \mathrm{O}_{2}+\% \mathrm{Ar}$ mixtures can be found in CALTECH detonation database [26] and in the Radulescu's dissertation [14]. Unfortunately, the cell size data for mixtures with $30 \%$ and $40 \%$ argon dilution are not available and therefore, those values are estimated by 
interpolation from the available data of other argon dilution percentage. The cell size correlations as a function of initial pressure taken from [14, 26] for different amounts of Ar dilution are reproduced in Table 2, and the corresponding plot is shown in Fig. 6.

Figure 7 illustrates the behavior between the critical tube diameter and the cell size in $\mathrm{C}_{2} \mathrm{H}_{2}+2.5 \mathrm{O}_{2}$ mixtures diluted with argon varied from 0 to $70 \%$. For stoichiometric acetylene-oxygen mixtures without and with small argon dilution (i.e., $0 \%$ and $22 \%$ ), the critical tube diameter is found to be closely about 13 times the detonation cell size $\lambda$. Taken into account the uncertainty of cell size values, this indeed agrees reasonably well with the classical empirical correlation of $d_{\mathrm{c}}=13 \lambda$. The correlation $d_{\mathrm{c}}$ $=13 \lambda$ still holds for mixtures with argon dilution of $30 \%$. When the argon dilution reaches $40 \%$, the proportionality factor between critical tube diameter and cell size gradually increases. Argon dilution up to $65 \%$ and $70 \%$, exhibit factor increases close to 25 , which is in good agreement with the results from [12]. As shown in Fig. 7, this transitional behavior in $d_{c} / \lambda$ appears to be rather abrupt when the argon dilution reaches about $40-50 \%$. To look at the similar effect of increasing amount of argon dilution on the critical energy, explosion length $R_{0}$ is given for further discussion. Explosion length is a characteristic length scale for blast waves, which is expressed by $R_{0}=\left(E_{\text {spherical }} / p_{0}\right)^{1 / 3}$ for the spherical geometry $[1,9,27]$, where $E_{\text {spherical }}$ is the point source energy and $p_{0}$ is the initial pressure. In other words, the explosion length can be used as an alternative expression of the critical energy. In the study of Zhang et al. [10], it was confirmed using different hydrocarbon mixtures that the relationship between explosion length and cell size was closely given by $R_{0} \approx 26 \lambda$ for the unstable detonations, while the proportionality factor increases in the stable detonations. For instance, Fig. 8 shows $R_{0} / \lambda$ obtained using the experimental curve fits as a function of different amount of argon dilution with the initial pressure of $10 \mathrm{kPa}$ and $15 \mathrm{kPa}$, respectively. One can see that $R_{0} \approx 26 \lambda$ holds for mixtures with argon dilution less than $40 \%$; and when argon dilution increases further above $50 \%$, the ratios $R_{0} / \lambda$ vary to $36.52,43.34$ and 
56.53 , subsequently. The change in $R_{0} / \lambda$ appears to be abrupt as argon dilution reaches up to 40 - $50 \%$, which agrees with the observation of $d_{c} / \lambda$ behavior.

The breakdown of both $d_{\mathrm{c}}=13 \lambda$ and $R_{0} \approx 26 \lambda$ relationships in mixtures when argon dilution is above 40 - 50\% suggests that there is a transition in the detonation dynamics. Indeed, this critical amount of argon dilution agrees approximately with experiments in porous tubes where evidences show that there is also a distinctive change in the failure mechanism near approximately $50-60 \%$ argon dilution $[4,14]$. This critical argon dilution corresponds roughly to the limit between regular and irregular cell structures, where highly regular cells were observed above the same degree of argon dilution [11, 29]. Studies suggest that below this amount of argon dilution, the cellular detonation front remains highly unstable and the cellular instabilities play a dominant role in the self-sustained propagation [14, 16, 30]. For explosive mixtures with argon dilution more than $50 \%$, the detonation becomes stable and regular - in the sense that the role of cellular instabilities are less prominent in the propagation mechanism of stable detonations in these mixtures. Consequently the failure and re-initiation mechanism for both the detonation limits and critical tube diameter phenomena are also different.

Figure 9 shows the correlation between critical tube diameter and critical initiation energy for direct initiation of spherical detonations in stoichiometric acetylene-oxygen mixtures diluted with different amount percentage of argon. It should be noted that the critical energies are measured at same critical initial pressures found in the critical tube diameter experiments. Equivalently, the critical tube diameter and critical energy are experimentally measured simultaneously at the same mixture sensitivity controlled by the initial pressure $p_{0}$. Although there exist several semi-empirical correlations [31, 32] or theoretical models to estimate the critical energy for direct initiation [e.g., 33-36], to compare the experimental results with theoretical predictions here we focus on an initiation model proposed by Lee 
$[31,37]$ which relates specifically the two measured quantities in this work, namely the critical initiation energy $E_{\mathrm{c}}$ and the critical tube diameter $d_{\mathrm{c}}$ as the length scale.

The surface energy model is a semi-empirical phenomenological model; its formulation originates from the relationship between direct initiation and the critical tube diameter phenomena. The link is established based on the minimum surface energy of the critical tube to the surface area of the critical size of the minimum detonation kernel, in other words, the correlation is through equating the surface energy contained in the wave in both cases at criticality. Thus the surface energy contained in the point blast initiated spherical detonation wave at the time when the wave has decayed to the CJ state from its overdriven state is equivalent to the energy in the planar detonation wave in the critical tube diameter situation. Hence equating the minimum surface areas of both waves at criticality:

$$
4 \pi R^{* 2}=\left(\frac{\pi}{4}\right) d_{c}^{2}
$$

Thus yields a kernel radius $R^{*}=\frac{1}{4} d_{c}$. From strong blast wave theory, the blast wave energy is given by:

$$
E_{c}=4 \pi I \gamma \quad p_{0} M_{s}^{2} R_{s}^{3}
$$

According to Zel'dovich's criterion [38], when the blast wave decays to $M_{\mathrm{s}}=M_{\mathrm{CJ}}$, it should engulf a kernel size of radius $R_{\mathrm{s}}=R^{*}$. Hence, this model leads to an expression linking the critical initiation energy and critical tube diameter [15, 31, 37]:

$$
E_{c}=4 \pi \gamma p_{0} M_{C J}^{2} I\left(\frac{d_{c}}{4}\right)^{3}
$$

where $p_{0}$ is the initial pressure of the mixture, and $M_{\mathrm{CJ}}$ is the mach number of the wave at CJ state. The numerical constant $I$ depends on the specific heat ratio $\gamma$ of the mixture. For $\gamma=1.4, I$ has a value of 0.423 for spherical geometry $[27,39]$. $d_{c}$ represents the critical tube diameter. As shown in Fig. 9, it appears that this scaling between the critical tube diameter and the critical energy in stoichiometric 
mixtures of acetylene-oxygen holds irrespectively of different degree of argon dilution and its relation is approximated reasonably well by the Eq. (3) based on the phenomenological model by Lee.

Chemical kinetic analysis is also performed to explain the effect of varying argon dilution on the dynamic parameters of acetylene-oxygen-argon detonations. The ZND detonation properties are computed using the CHEMKIN package [40] together with the San Diego chemical kinetic mechanism [41]. For the San Diego chemical kinetic mechanism, Varatharajan et al. [42] employed a new detailedchemistry description that involves 114 elementary steps among 28 chemical species, revising questionable rate parameters for certain elementary steps, notably concerning $\mathrm{OH}$ and $\mathrm{O}_{2}$ attack on $\mathrm{C}_{2} \mathrm{H}_{2}$. Various levels of description of acetylene ignition and detonation chemistry were generated for computational and theoretical studies of high-temperature ignition and detonation behavior of fuels that contain or produce appreciable amounts of acetylene. These have mostly been validated and optimized for detonation simulations in acetylene-oxygen-diluent mixtures [42].

The induction zone length variation with varying amount of argon dilution in stoichiometric acetylene-oxygen mixtures at different initial pressures is illustrated in Fig. 10. Here the induction length is determined as the length of the thermally neutral period in the ZND structure. It is defined as the distance from the leading shock to the maximum temperature gradient in the ZND temperature profile as used in previous studies [43]. As obtained from the experiments, both measured dynamic parameters increase consequently with an increase of argon dilution. This can be explained readily due to the fact that by increasing the amount of argon dilution, the ZND induction length which provides a chemical length scale of the detonation wave increases as shown in Fig. 10. For instance, as illustrated by the Zeldovich's criterion [38], the critical energy is of the cube of induction length for spherical detonation, i.e., $E_{\mathrm{c}} \sim \Delta_{\mathrm{I}}^{3}$. Furthermore, the critical tube diameter is well established to be proportional to the cell size, $d_{\mathrm{c}} \sim \lambda$ while chemical kinetic analysis often shows $\lambda$ can be scaled linearly with $\Delta$, hence resulting 
in $d_{\mathrm{c}} \sim \Delta$. Therefore, the results of the induction length analysis are in accord with the experimental observation that with larger amount of argon dilution, the increase in the chemical induction length leads to an increase of both critical tube diameter and direct initiation energy. It can also be seen from Fig. 10 that, for the mixture with the same argon dilution \%, the induction zone length at lower pressure is always larger than the higher pressure (i.e. $\Delta_{\mathrm{I}} \sim p_{0}{ }^{-1}$ ), which can thus render the critical tube diameter and critical initiation energy larger. There also seems to be a noticeable exponential increase in the ZND induction length with the increasing amount argon dilution (above 40-50\%). Intuitively, this may perhaps explain the onset of derivation of scaling laws shown in Figs. 7 and 8, i.e., $d_{\mathrm{c}}=13 \lambda$ and $R_{0}=$ $26 \lambda$, at this same amount of argon dilution. However, it is important to realize that $\lambda$ also increases with the induction length roughly at a similar rate and hence, the induction length plot alone cannot fully explain the breakdown of these scaling relationships.

Apart from the ZND induction length analysis, as discussed in previous work the effect of argon dilution can be better explained from the consideration of detonation stability [16, 30]. To provide further insight on the breakdown of the universal scaling laws (i.e., $d_{\mathrm{c}}=13 \lambda$ and $R_{0}=26 \lambda$ ) as the amount of argon dilution increases, we look at the variation of the stability parameter $\chi$. This stability parameter $\chi$ was previously proposed by considering different length scales and sensitivity of the chemical reaction. We define $\chi$ as the reduced effective activation energy of the induction zone $\varepsilon_{\mathrm{I}}$ multiplied by the ratio of induction zone $\Delta_{\mathrm{I}}$ to the exothermic heat release length $\Delta_{\mathrm{R}}$, i.e.,

$$
\chi=\varepsilon_{I} \frac{\Delta_{I}}{\Delta_{R}}=\varepsilon_{I} \Delta_{I} \frac{\dot{\sigma}_{\max }}{u_{C J}^{\prime}}
$$

Again, $\varepsilon_{I}$ is the reduced activation energy with respect to the shock temperature $T_{\mathrm{s}}$ describing the sensitivity of the thermally neutral induction process (i.e., $\varepsilon_{\mathrm{I}}=E_{\mathrm{a}} / R T_{\mathrm{s}}$ where $R$ is the ideal gas constant). 
This can be obtained by constant-volume explosion calculations. Assuming that the induction time $\tau_{\mathrm{i}}$ has an Arrhenius form:

$$
\tau_{i}=A \rho^{n} \exp \left(\frac{E_{a}}{R T}\right)
$$

The reduced activation energy $E_{\mathrm{a}} / R T_{\mathrm{s}}$ can be determined by

$$
\varepsilon_{I}=\frac{E_{a}}{R T_{s}}=\frac{1}{T_{s}} \frac{\ln \tau_{2}-\ln \tau_{1}}{\frac{1}{T_{2}}-\frac{1}{T_{1}}}
$$

where two constant-volume explosion simulations are run with initial conditions $\left(T_{1}, \tau_{1}\right)$ and $\left(T_{2}, \tau_{2}\right)$. Conditions for states one and two are obtained by considering the effect of a change in the shock velocity by $\pm 1 \% D_{\text {CJ. }}[44]$.

The heat release length $\Delta_{\mathrm{R}}$ is estimated by using the maximum thermicity $\dot{\sigma}_{\max }$ multiplied by the CJ particle velocity in shock-attached frame $u_{C J}^{\prime}$ [30]. For the thermicity $\dot{\sigma}$, it is defined in Fickett and Davis [45] as:

$$
\dot{\sigma}=\sum_{i=1}^{N_{s}}\left(\frac{W}{W_{i}}-\frac{h_{i}}{C_{p} T}\right) \frac{d Y_{i}}{d t}
$$

where $W$ is the mean molar mass of the mixture, $C_{\mathrm{p}}$ is the mixture specific heat at constant pressure, and $h_{\mathrm{i}}$ is the specific enthalpy of specie $i$. All these parameters can be obtained from the chemical kinetic calculation using the CHEMKIN package [40].

Unstable detonations with highly irregular cellular structure as in most undiluted combustible mixtures usually have a high degree of instability characterized by a large value of $\chi$. As noted by Lee [1], detonations characterized by a high degree of instability, i.e., with irregular cellular structures and strong transverse waves, are usually more robust than stable detonations with very regular, stable structure. Unstable, irregular mixtures are perhaps more prompt to the formation of localized explosions, 
which may thus facilitate or ease the initiation or the onset of a detonation. The variation of the stability parameter $\chi$ with increasing amount of argon dilution of acetylene-oxygen-argon detonations at different initial pressures is shown in Fig. 11. It can be seen that the value of stability parameter $\chi$ decreases with the increase of argon dilution. In other words, high degree of argon dilution has a stabilizing effect on the detonation wave, which decreases the detonation sensitivity [16]. It is possible to observe from this plot a slightly faster decrease in $\chi$ when the amount of argon dilution passes $40 \%$. Although a significant abrupt change cannot be seen in the stability parameter $\chi$ at around $40 \%$ argon dilution as in the ZND induction length plot, the result perhaps suggests that the 2-D neutral stability boundary may locate around the value of $\chi$ at this critical amount of dilution. However, more stability analysis is required to further confirm this conclusion. In fact, the degree of cell regularity of the detonation also may not be linearly proportional to the value of $\chi$. Nevertheless, our previous studies [16, 30] on this parameter $\chi$ by comparing the detonation cellular patterns of different mixtures indicate that below the same critical $\chi$ as obtained for $40 \%-50 \%$ argon dilution, the detonation wave is more stable with increasing regularity in cellular pattern. After all, the breakdown of different classical correlations valid for most undiluted hydrocarbon mixtures, as well as the increase in critical tube diameter and critical energy for mixtures diluted with an increasing amount of argon, are evidenced by both the ZND induction length analysis and the concept of detonation stability characterized by the parameter $\chi$.

\section{Concluding Remarks}

In this investigation, critical tube diameter and critical energy for direct initiation of spherical detonations were measured for stoichiometric acetylene-oxygen mixtures diluted with varying amount of argon at different initial pressures. It is shown that the critical tube diameter and critical initiation energy increase gradually with the increase of argon dilution. The effect of argon dilution on the scaling 
between different dynamic detonation parameters was then investigated. By comparing the critical tube diameter with the available cell size data in literature, it is found that the classical $d_{\mathrm{c}}=13 \lambda$ relationship holds for $0 \%-30 \%$ argon diluted mixtures; while increasing the amount of argon, the proportionality factor approaches 25 with $70 \%$ argon dilution. Similar observation is obtained for the explosion length scaling of which the correlation $R_{0}=26 \lambda$ breaks down when argon dilution reaches $40 \%$ or above. The change appears to be abrupt and the transition is thought to be due to dynamic effects in the detonation behavior, i.e., the detonation remains unstable and cellular instabilities play a dominant role in the selfsustained propagation of the detonation in mixtures without or with small amount of argon dilution. For cases with increasing argon dilution (e.g., above 40 - 50\%) the detonation structure becomes regular and its propagation subsequently relies on the classical ZND shock ignition mechanism. Regardless of the amount of argon dilution, the critical tube diameter and critical energy can still be scaled adequately with each other and the phenomenological model based on the surface energy concept proposed by Lee appears to be valid for all stoichiometric acetylene-oxygen mixtures diluted with different amount of argon ranging from $0 \%$ to $70 \%$. The ZND analysis with chemical kinetics and stability consideration both provide important aspects to elucidate the effect of argon dilution on the trend of critical tube diameter as well as critical initiation energy variation.

\section{Acknowledgments}

This work is supported by Fundamental Research Funds for the Central Universities (Grant No.: 222201314030), Shanghai Postdoctoral Sustentation Fund, China (Grant No.:13R21411800), Postdoctoral Science Foundation of China (Grant No.: 2012M520852), and the Natural Sciences and Engineering Research Council of Canada (NSERC). 


\section{References}

[1] J. H. S. Lee, The detonation phenomenon. Cambridge University Press: Cambridge, 2008.

[2] G. Dupré, O. Peraldi, J. H. Lee, R. Knystautas, Progress in Astronautics and Aeronautics 114 (1988) 248-263.

[3] A. Teodorczyk, J. H. S. Lee, Shock Waves 4 (4) (1995) 225-236.

[4] M. I. Radulescu, J. H. S. Lee, Combust Flame 131 (1-2) (2002) 29-46.

[5] A. Camargo, H. D. Ng, J. Chao, J. H. S. Lee, Shock Waves 20 (6) (2010) 499-508.

[6] J. H. S. Lee, Dynamics of Exothermicity (1995) 321--335.

[7] V. V. Mitrofanov, R. I. Soloukhin, Sov. Phy. Dokl 9 (12) (1965) 1055-1058.

[8] R. Knystautas, J. H. Lee, C. M. Guirao, Combust Flame 48 (1982) 63-83.

[9] M. I. Radulescu, A. J. Higgins, J. H. S. Lee, S. B. Murray, Proc. Combust. Inst 28 (1) (2000) 637644.

[10] B. Zhang, H. D. Ng, J. H. S. Lee, Shock Waves 22 (2012) 275 - 279.

[11] J. E. Shepherd, I. Moen, S. Murray, P. A. Thibault, Proc. Combust. Inst 21 (1986) 1649-1658.

[12] D. Desbordes, C. Guerraud, L. Hamada, H. N. Presles, Prog. Astronaut. Aeronaut 153 (1993) 347359.

[13] I. O. Moen, A. Sulmistras, G. Thomas, D. Bjerketvedt, P. A. Thibault, Prog. Astronaut. Aeronaut 106 (1986) 220-243.

[14] M. I. Radulescu. The propagation and failure mechanism of gaseous detonation: experiments in porous-walled tubes. McGill University, Montreal, 2003.

[15] B. Zhang, V. Kamenskihs, H. D. Ng, J. Lee, Proc. Combust. Inst 33 (2) (2010) 2265-2271.

[16] M. I. Radulescu, H. D. Ng, J. H. S. Lee, B. Varatharajan, Proceedings of the Combustion Institute 
29 (2) (2002) 2825-2831.

[17] J. Chao, H. D. Ng, J. H. S. Lee, Proceedings of the Combustion Institute 32 (2) (2009) 2349-2354.

[18] R. Klein, J. C. Krok, J. E. Shepherd, Curved quasi-steady detonations: asymptotic analysis and detailed chemical kinetics; California Institute of Technology: 1995.

[19] J. Yao, D. S. Stewart, Combust Flame 100 (4) (1995) 519 - 528.

[20] V. Kamenskihs, H. D. Ng, J. H. S. Lee, Combust Flame 157 (9) (2010) 1795-1799.

[21] B. Zhang, H. D. Ng, R. Mével, J. H. S. Lee, Int J Hydrogen Energy 36 (9) (2011) 5707-5716.

[22] B. Zhang, H. D Ng, J. H. S. Lee, J Loss Prevent Proc (2013) In press,

(http://dx.doi.org/10.1016/j.jlp.2013.07.011)

[23] B. Zhang, C. H. Bai, Safety Sci 53 (2013) 153-159.

[24] B. Zhang, H. D. Ng, J. H.S. Lee, Shock Waves 22 (1) (2012) 1-7.

[25] B. Zhang, H. D. Ng, J. H. S. Lee, Combust Flame 159 (9) (2012) 2944-2953.

[26] M. Kaneshige, J. E. Shepherd Detonation database; GALCIT Report FM97-8, California Institute of Technology, Pasadena, CA.: 1997.

[27] J. H. S. Lee, Ann. Rev. Phys. Chem 28 (1977) 75-104.

[28] S. B. Murray, J. H. S. Lee, Pro. Astro. Aero. 94 (1984) 80-103.

[29] M. Vandermeiren, P. J. Van Tiggelen, Prog. Astronaut. Aeronaut 94 (1984) 104-117.

[30] H. D. Ng, M. I. Radulescu， A. J. Higgins, N. Nikiforakis, J. H. S. Lee, Combust Theor Model 9 (3) (2005) $385-401$.

[31] W. B. Benedick, C. M. Guirao, R. Knystautas, In Prog. Astronaut. Aeronaut 106 (1986) 181-202.

[32] J. Brossard, I. Sochet, J Phys IV 10 (11) (2000) 81-89.

[33] L. He, P. Clavin, J Fluid Mech 277 (1994) 227-248.

[34] A. R. Kasimov, D. S. Stewart, Theory of detonation initiation and comparison with experiment, 
Report \#1035, Theoretical \& Applied Mechanics, University of Illinois at Urbana-Champaign, 2004.

[35] C. A. Eckett, J. J. Quirk, J. E. Shepherd, J Fluid Mech 421 (2000) 147 - 183.

[36] H. Soury, K. Mazaheri, Int J Hydrogen Energy, 34 (2009) 9847-9856.

[37] J. H. S. Lee, R. Knystautas, C. M. Guirao, in: Fuel - Air Explosions, University of Waterloo Press: Waterloo, 1982; pp 157-187.

[38] Y. B. Zeldovich, S. M. Kogarko, N. N. Simonov, Sov. Phys. Tech. Phys 1 (8) (1956) 1689--1713.

[39] G. G. Bach, J.H.S. Lee, AIAA J. 7 (1969) 742-744

[40] R. J. Kee, F. M. Rupley, J. A. Miller Sandia National Laboratories report SAND89-8009; SAND89-8009; USA: 1989.

[41] San Diego Mechanism web page, Mechanical and Aerospace Engineering (Combustion Research), University of California at San Diego (http://combustion.ucsd.edu).

[42] B. Varatharajan, F. A. Williams, Combust Flame 124 (2001) 624-645.

[43] J. E. Shepherd, Prog Astro Aeronaut 106 (1986) 263-293.

[44] E. Schultz, J. E. Shepherd, Validation of detailed reaction mechanisms for detonation simulation. GALCIT Technical Report FM99-5, California Institute of Technology, Pasadena, CA.: 2000.

[45] W. Fickett, W. C. Davis, Detonation. University of California Press: Berkeley, 1979. 


\section{Table}

Table 1 Initial conditions used in the critical tube diameter experiment

Table 2 The cell size correlation for $\mathrm{C}_{2} \mathrm{H}_{2}+2.5 \mathrm{O}_{2}+\%$ Ar mixtures as a function of initial pressure given by: $\lambda[\mathrm{mm}]=C \cdot\left(p_{o}[\mathrm{kPa}]\right)^{-\mu}$ (Parameters taken from [14]). 


\begin{tabular}{cc}
\hline Ar,\% & $p_{0}(\mathrm{kPa})$ \\
\hline 0 & $11-21$ \\
22 & $13-27$ \\
30 & $16-33$ \\
40 & $25-51$ \\
50 & $43-81$ \\
65 & $55-99$ \\
70 & $75-141$ \\
\hline
\end{tabular}

Table 1. 


\begin{tabular}{ccc}
\hline Ar, \% & $C$ & $\mu$ \\
\hline 0 & 28.7 & 1.26 \\
22 & 39.6 & 1.21 \\
50 & 61.5 & 1.12 \\
65 & 93.1 & 1.20 \\
70 & 113.8 & 1.20 \\
75 & 152.0 & 1.15 \\
81 & 367 & 1.23 \\
\hline
\end{tabular}

Table 2. 


\section{Figure Captions}

Fig. 1 Schematics of the experimental setup for a) the critical tube diameter; and b) direct initiation experiments

Fig. 2 Arrival time trace of a planar detonation emerging into an unconfined space: successful initiation of a spherical detonation in stoichiometric $\mathrm{C}_{2} \mathrm{H}_{2}+2.5 \mathrm{O}_{2}$ mixture at an initial pressure of $12 \mathrm{kPa}$ [22]

Fig. 3 Arrival time trace of a planar detonation emerging into an unconfined space: unsuccessful initiation of a spherical detonation in stoichiometric $\mathrm{C}_{2} \mathrm{H}_{2}+2.5 \mathrm{O}_{2}$ mixture at an initial pressure of $11 \mathrm{kPa}$ [22]

Fig. 4 Variation of the critical tube diameter with initial pressure for different amount of argon dilution in stoichiometric $\mathrm{C}_{2} \mathrm{H}_{2}+2.5 \mathrm{O}_{2}+\%$ Ar mixtures

Fig. 5 Variation of the critical energy for direct initiation with initial pressure for different amount of argon dilution in stoichiometric $\mathrm{C}_{2} \mathrm{H}_{2}+2.5 \mathrm{O}_{2}+\%$ Ar mixtures

Fig. 6 Cell size as a function of initial pressure in $\mathrm{C}_{2} \mathrm{H}_{2}+2.5 \mathrm{O}_{2}+\% \mathrm{Ar}[14,26]$

Fig. 7 Critical tube diameter as a function of cell size for varying amount of argon dilution in stoichiometric $\mathrm{C}_{2} \mathrm{H}_{2}+2.5 \mathrm{O}_{2}+\%$ Ar mixtures

Fig. $8 R_{0} / \lambda$ as a function of argon dilution in stoichiometric $\mathrm{C}_{2} \mathrm{H}_{2}+2.5 \mathrm{O}_{2}+\%$ Ar mixtures

Fig. 9 Critical tube diameter as a function of direct initiation energy for $\mathrm{C}_{2} \mathrm{H}_{2}+2.5 \mathrm{O}_{2}+\%$ Ar mixtures diluted with a) $0 \%$; b) 22\%; c) 30\%; d) 40\%; e) 50\%; f) 65\%; and g) 70\% argon

Fig. $10 \mathrm{ZND}$ induction zone length variation with varying amount of argon dilution in $\mathrm{C}_{2} \mathrm{H}_{2}+2.5 \mathrm{O}_{2}+$ \%Ar mixtures at three different initial pressures

Fig. 11 Stability parameter $\chi$ as a function of varying amount argon dilution in $\mathrm{C}_{2} \mathrm{H}_{2}+2.5 \mathrm{O}_{2}+\% \mathrm{Ar}$ mixtures at three different initial pressures 


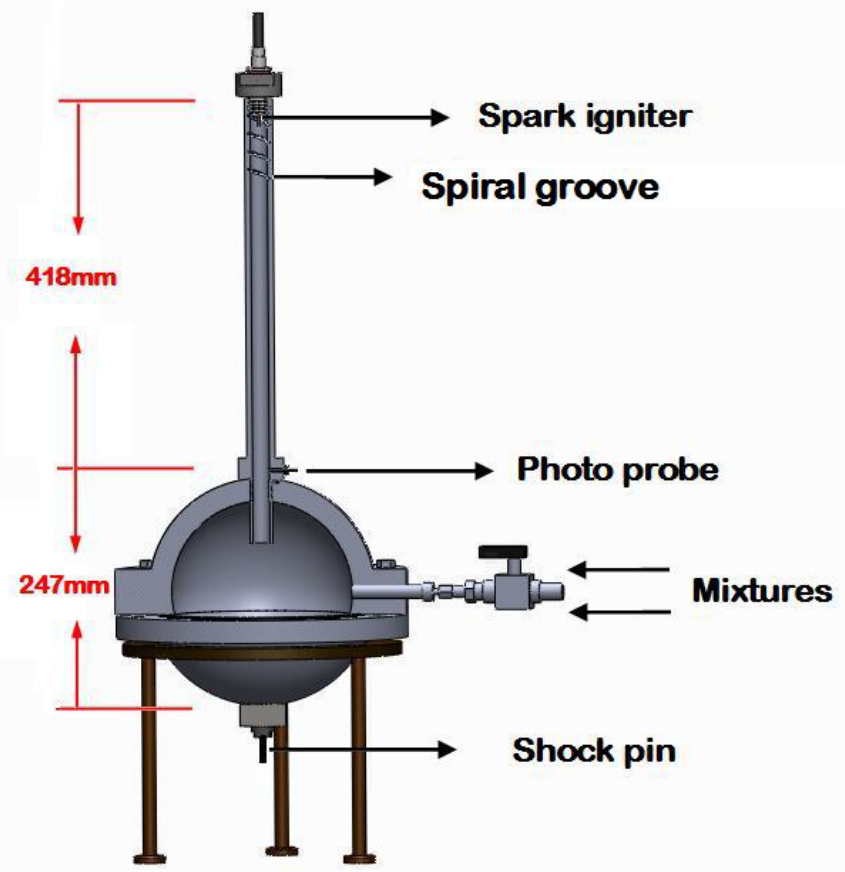

(a)

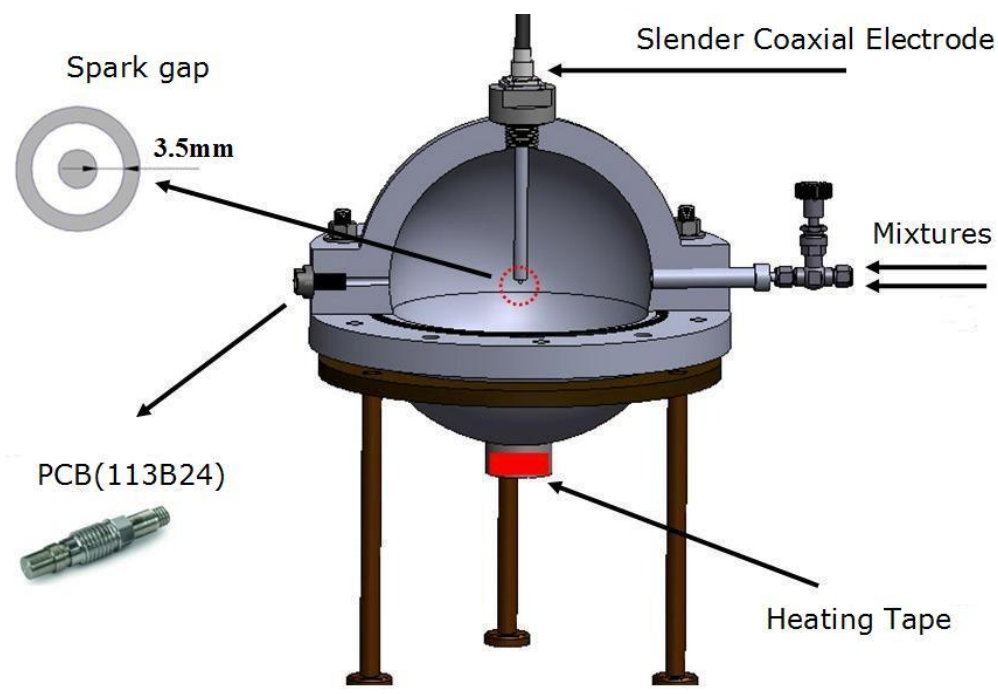

(b)

Fig. 1 


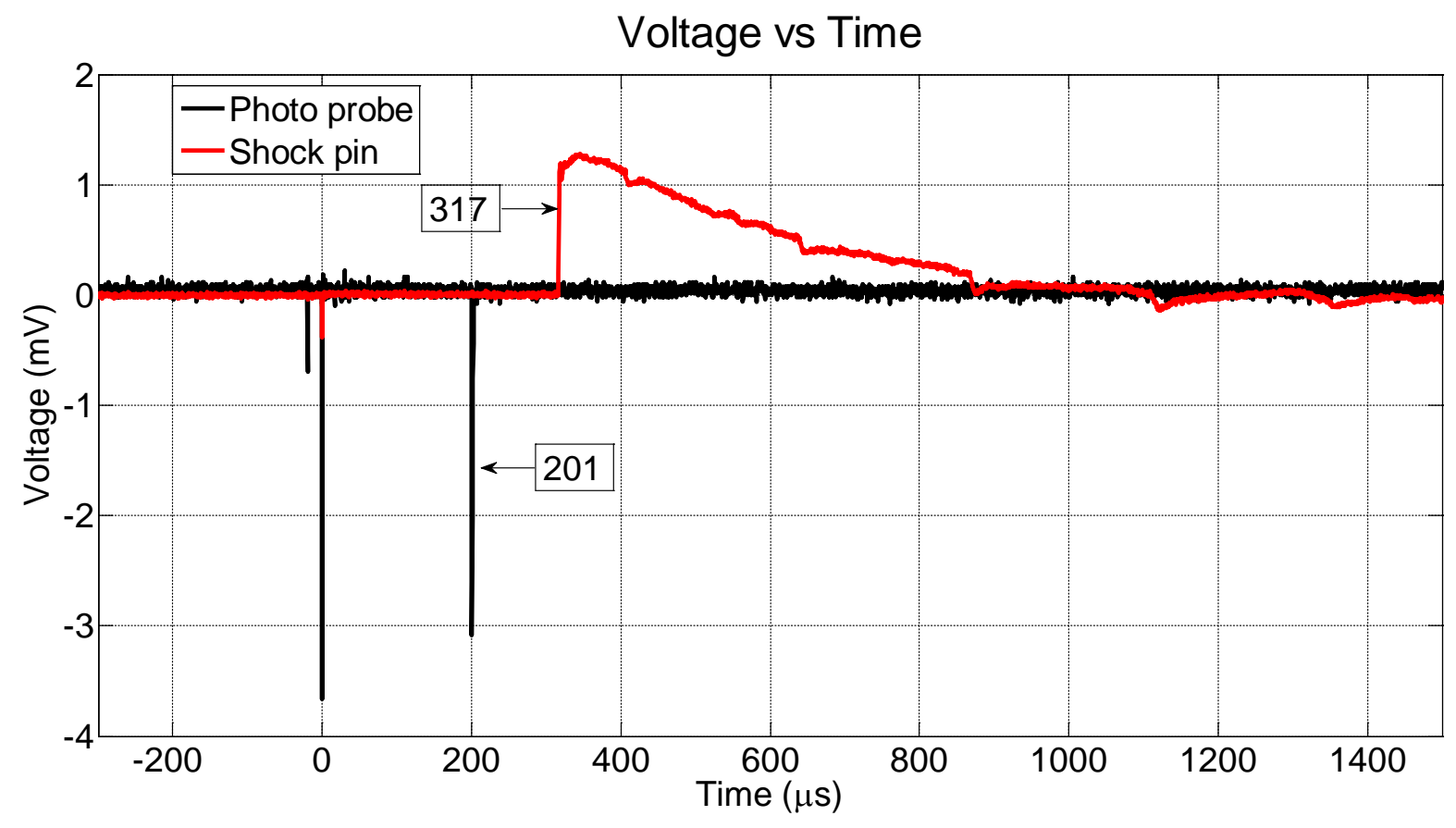

Fig. 2 


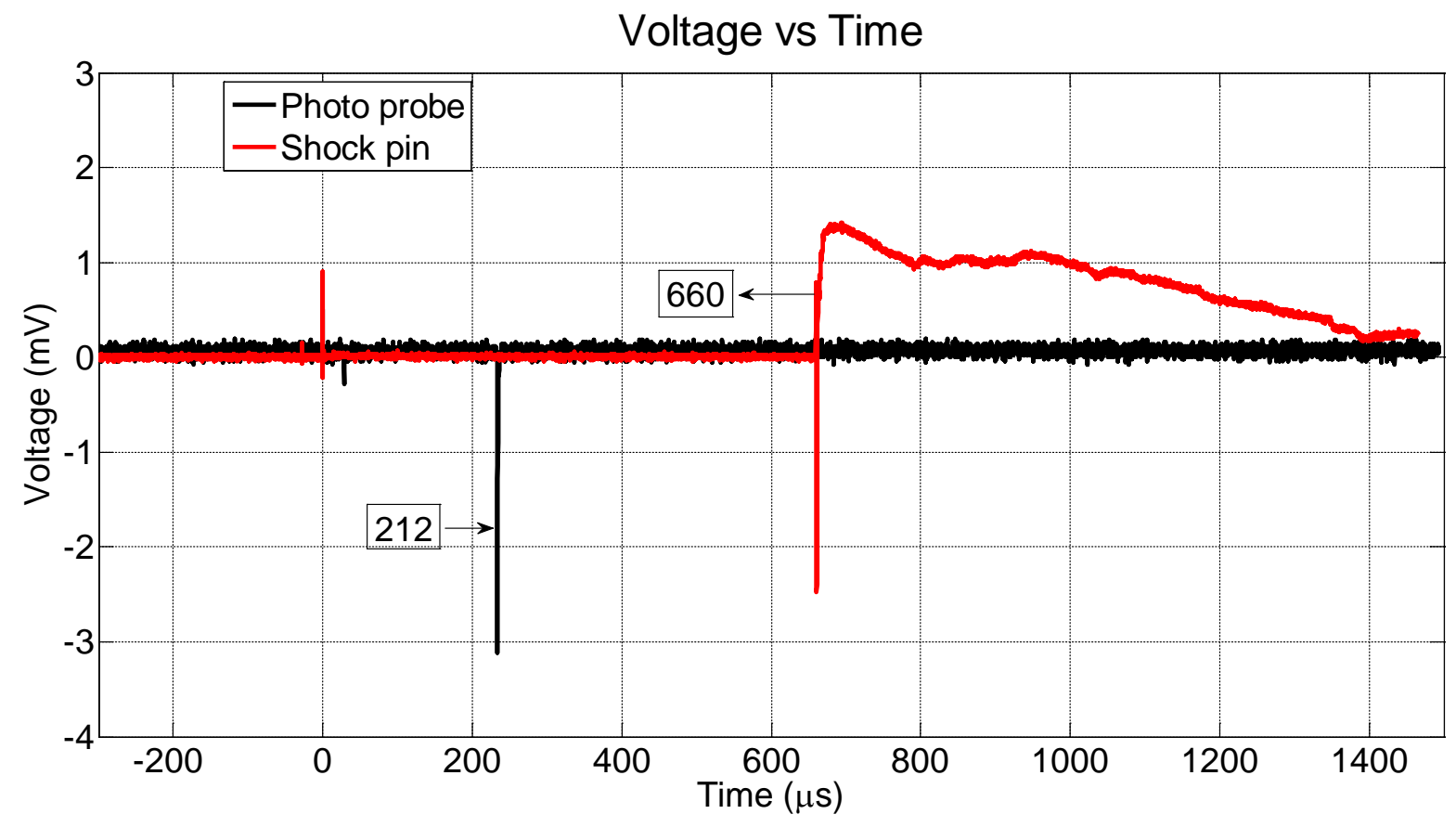

Fig.3 


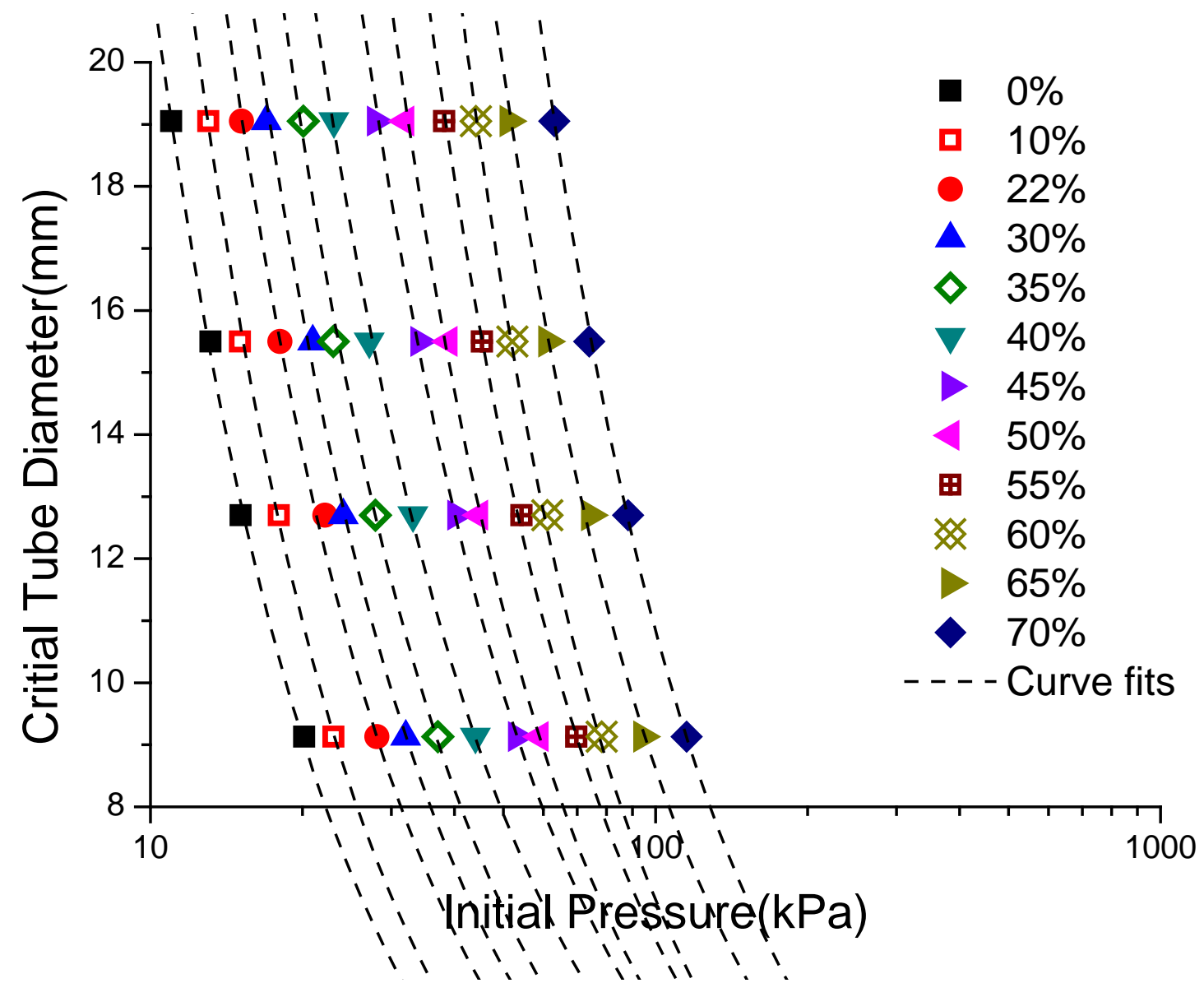

Fig. 4 


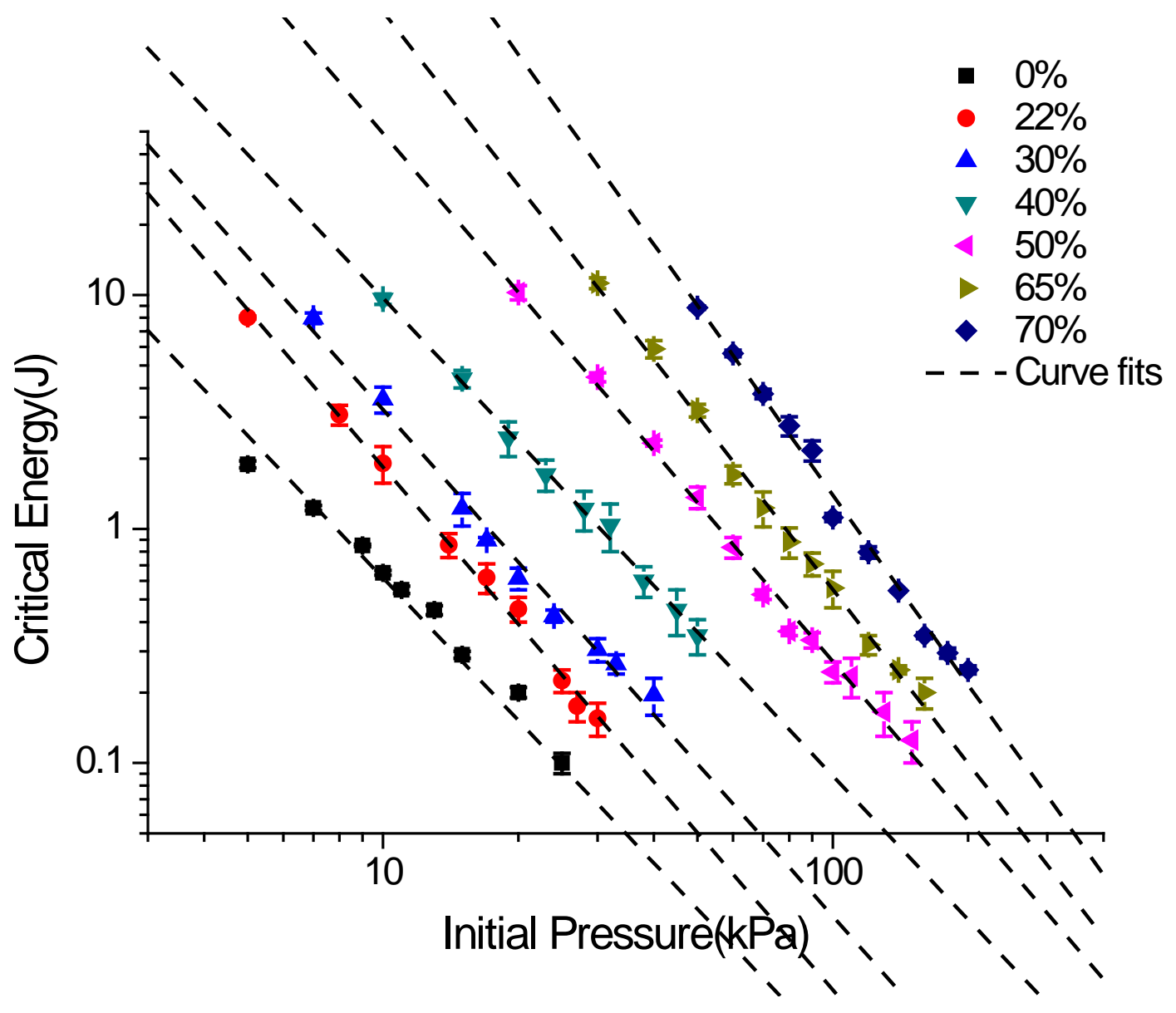

Fig. 5 


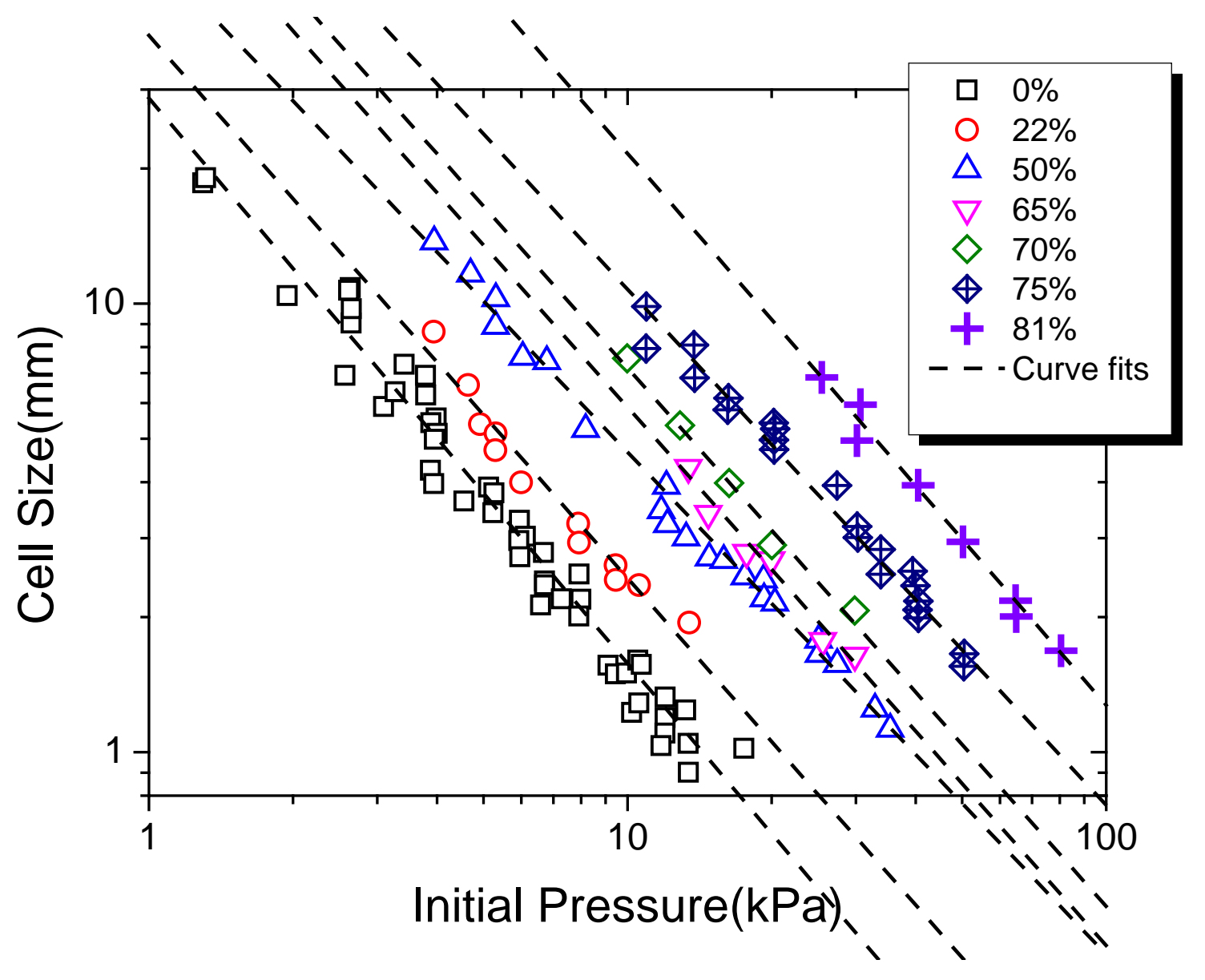

Fig. 6 


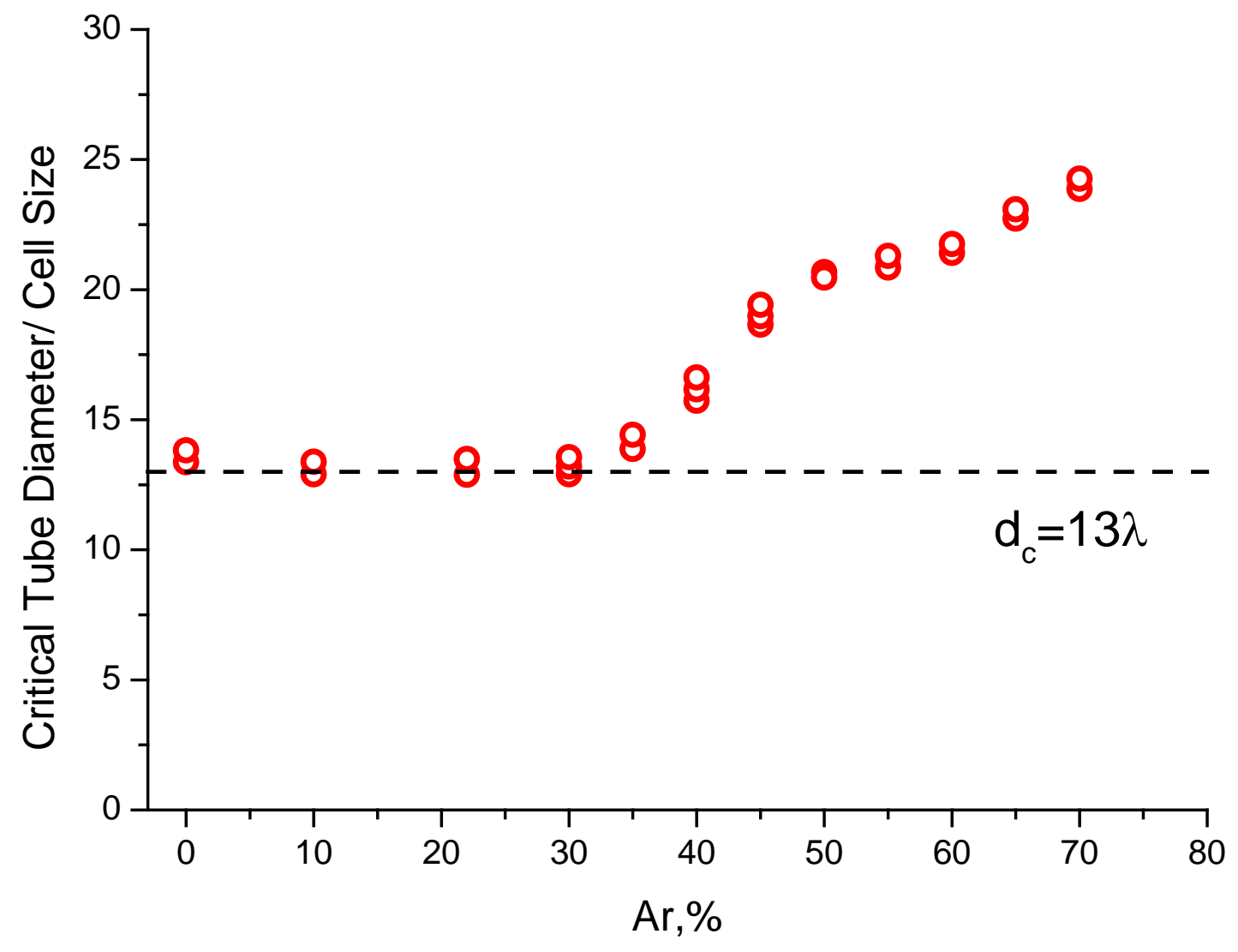

Fig. 7 


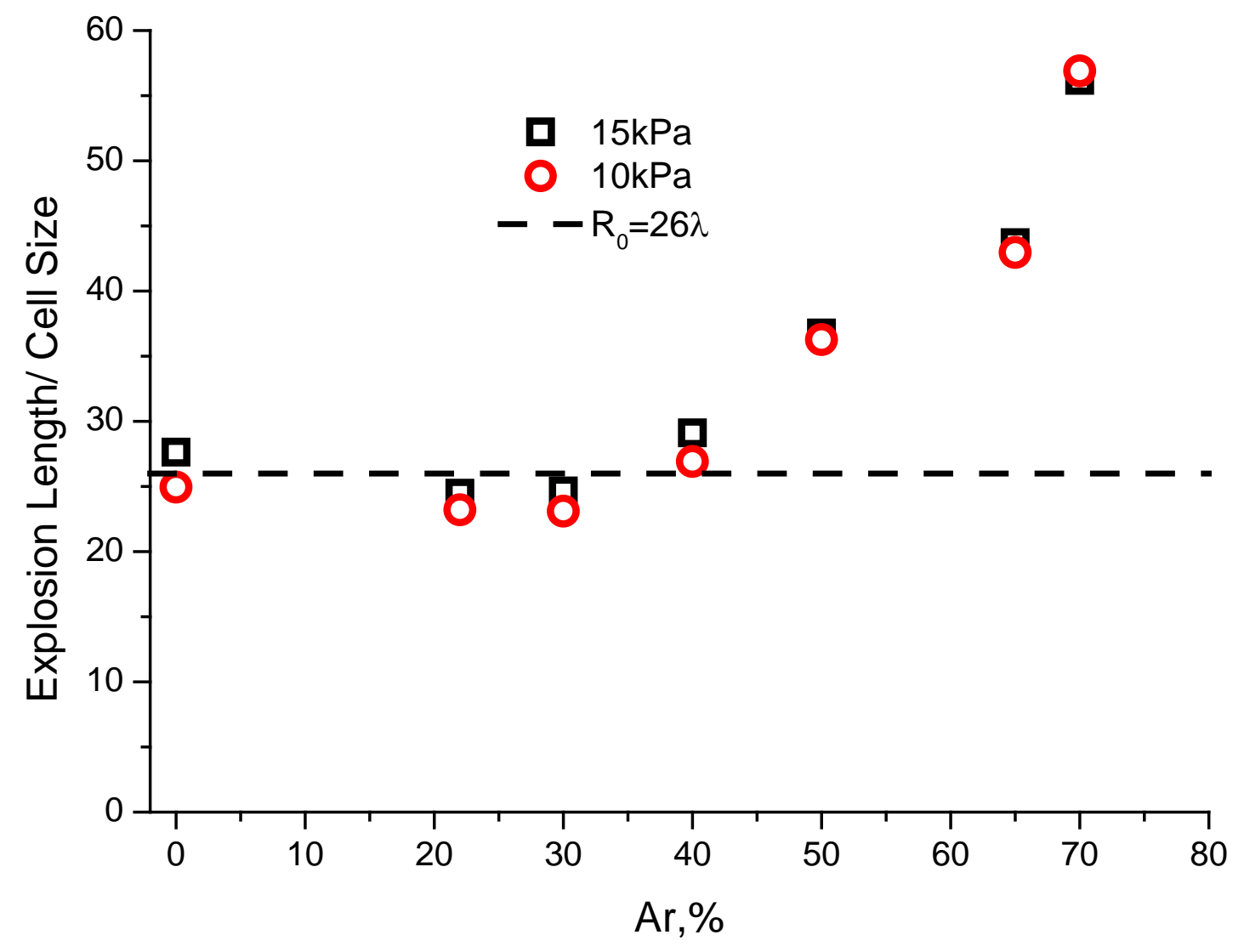

Fig.8 


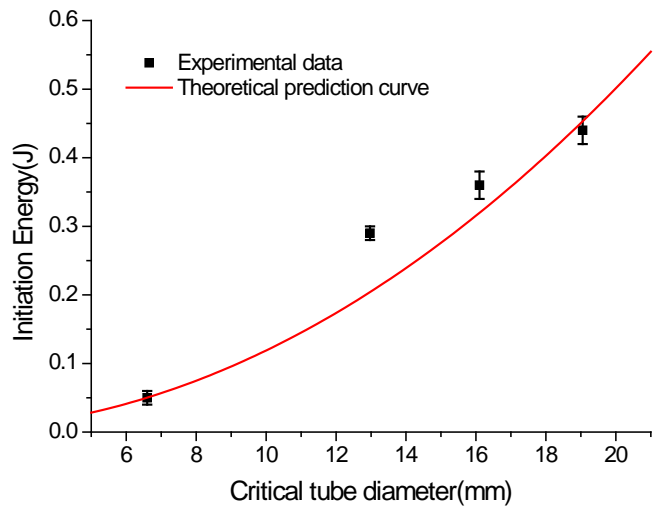

(a)

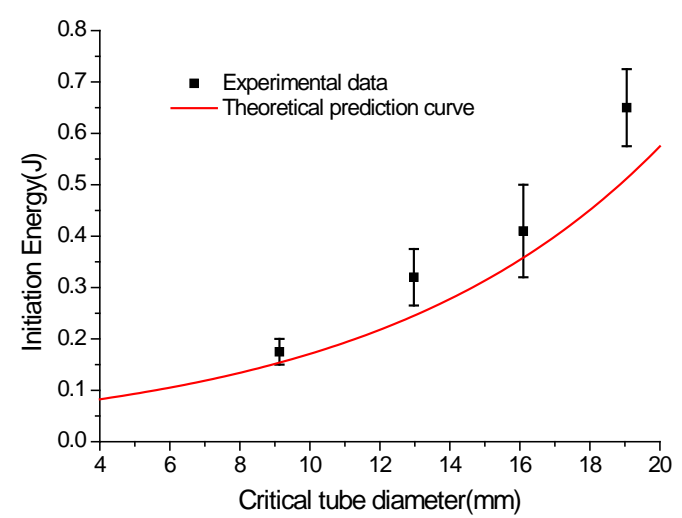

(b)

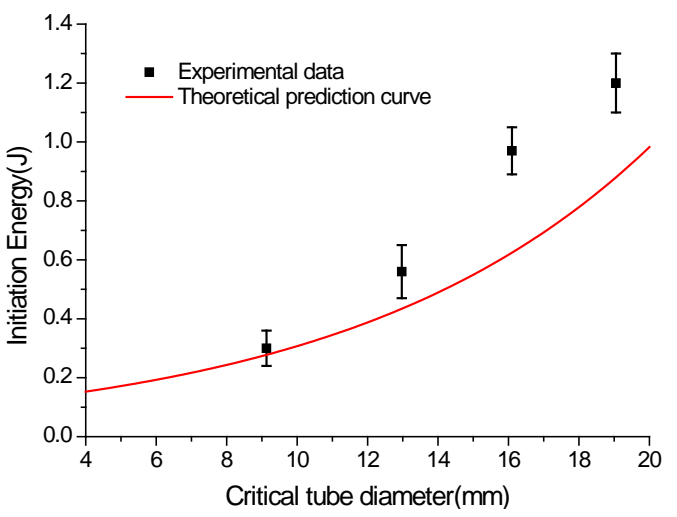

(d)

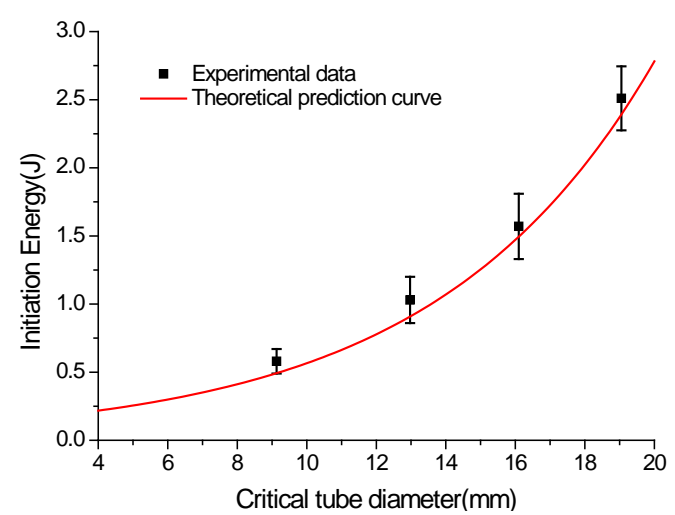

(f)

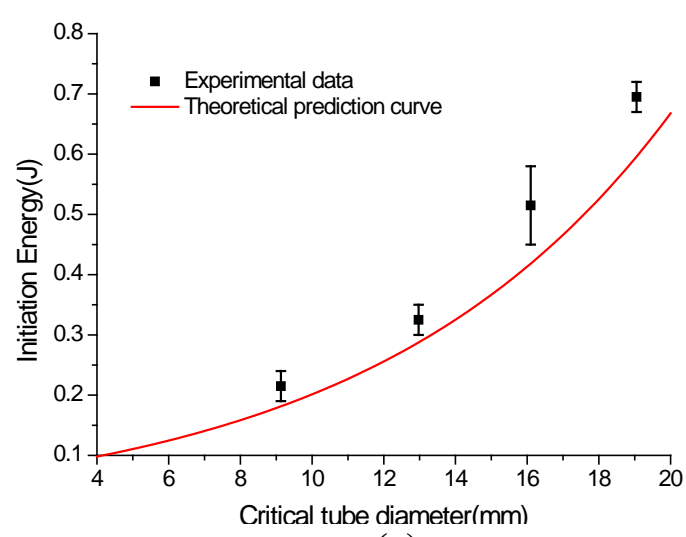

(c)

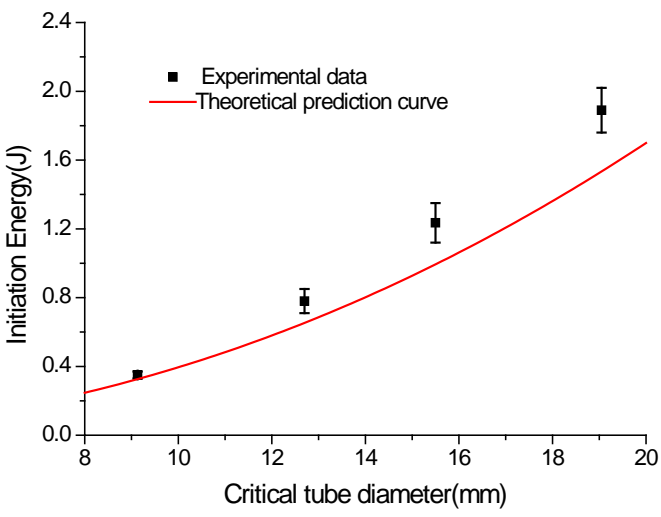

(e)

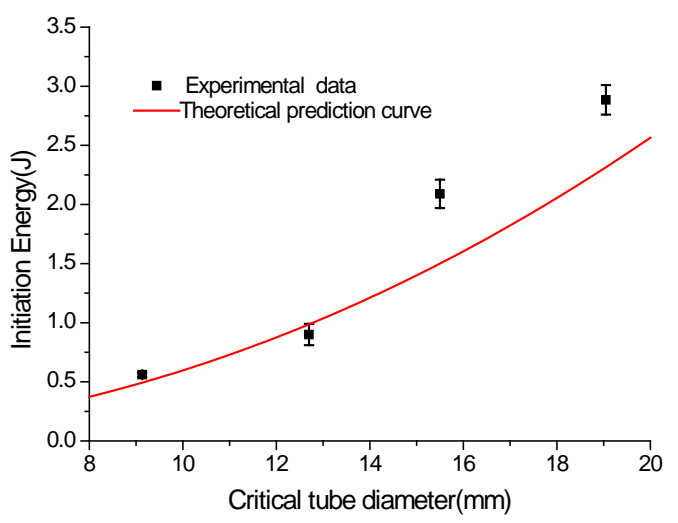

(g)

Fig. 9 


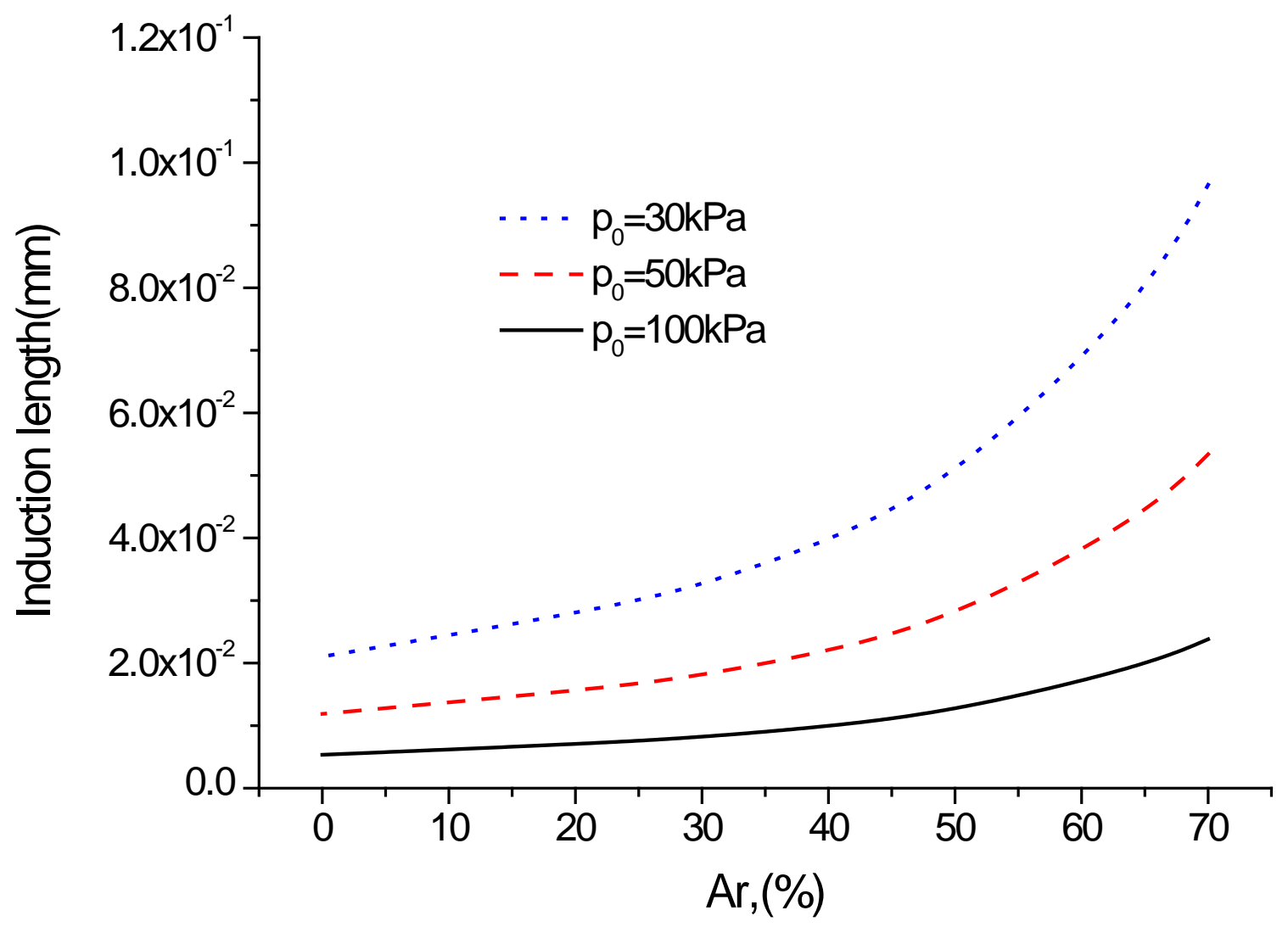

Fig. 10 


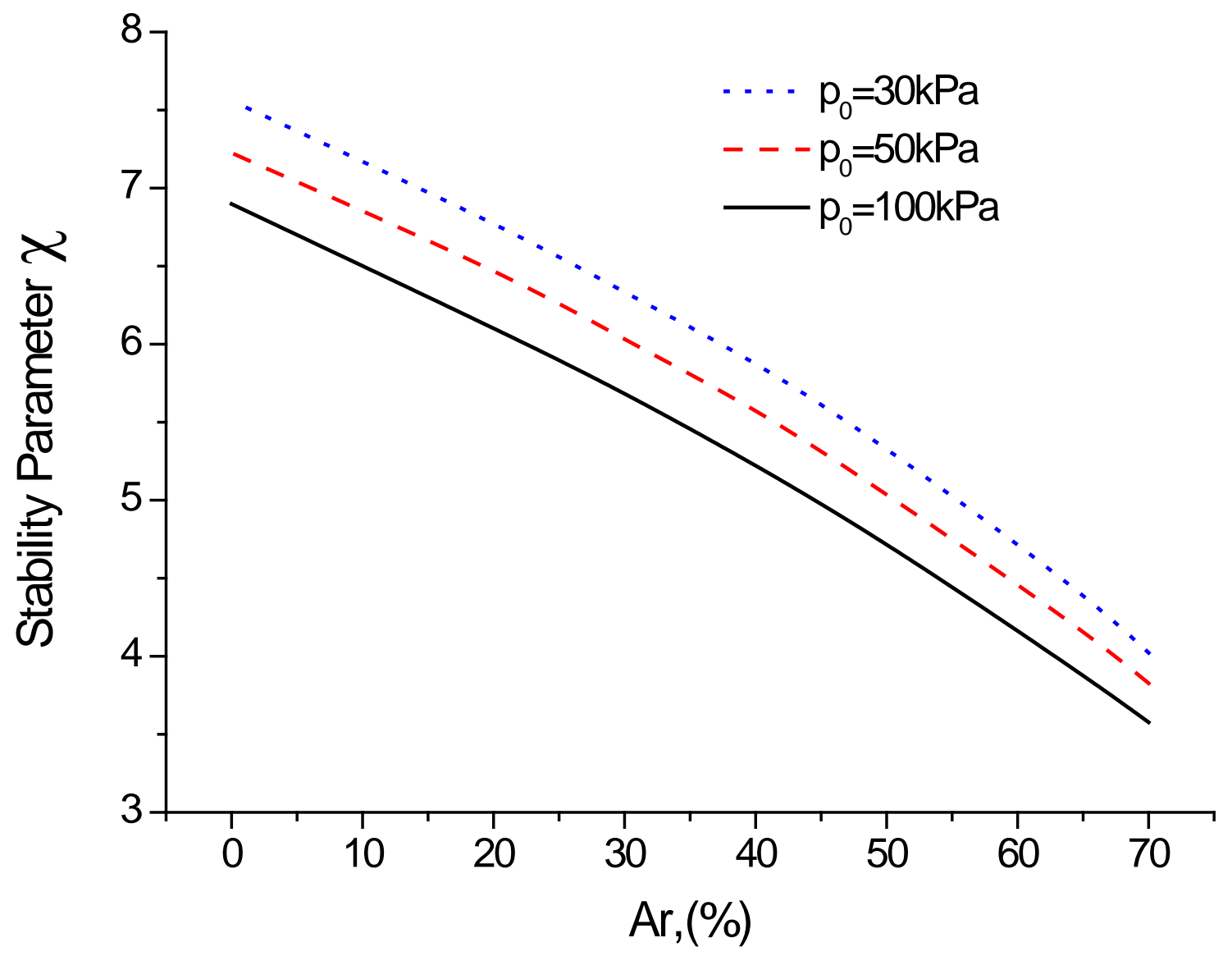

Fig. 11 